\title{
Effects of L-Carnitine Supplementation on MicroRNA-217 Expression Level in Non-alcoholic Steatohepatitis Suffering Patients with Obesity: A Randomized Controlled Trial
}

\section{Farzaneh Iravani}

Mashhad University of Medical Sciences

Fatemeh Roudi

Mashhad University of Medical Sciences

Shirin Amiri-Moghadam

Neyshabur University of Medical Sciences

Mahsa Asle-Mohajeri

Mashhad University of Medical Sciences

Maryam Salehi

Mashhad University of Medical Sciences

Mohsen Azimi-Nezhad

Neyshabur University of Medical Sciences

Majid Mojarrad

Mashhad University of Medical Sciences

Mohsen Nematy ( $\nabla$ Mohsennematy110@gmail.com )

Mashhad University of Medical Sciences

\section{Research Article}

Keywords: Nonalcoholic Fatty Liver Disease, Nonalcoholic Steatohepatitis, MicroRNA, L-Carnitine

Posted Date: January 21st, 2021

DOI: https://doi.org/10.21203/rs.3.rs-144328/v1

License: (c) (i) This work is licensed under a Creative Commons Attribution 4.0 International License. Read Full License 


\section{Abstract}

\section{Background}

Nonalcoholic steatohepatitis (NASH) is a severe subtype of Nonalcoholic fatty liver disease (NAFLD) and a progressive chronic liver disease which is associated with oxidative stress, inflammation, fibrosis, lipotoxicity, and altered mitochondrial metabolism. Silent Information Regulator 1 (SIRT1) gene is considered as a potential therapeutic target in NAFLD, microRNA-217 (MiRNA-217) inhibits expression of SIRT1 and this inhibition may play role in the pathogenesis of NASH. On the other hand, L-carnitine may have beneficial effects on NASH. Therefore, we investigated the effect of L-carnitine on the MiRNA-217 expression levels in obese NASH suffering patients.

\section{Methods}

This double-blind randomized controlled trial (RCT) was carried out in the nutrition clinic of Mashhad University of Medical Sciences (March 2013-June 2014). Seventy-one obese NASH suffering patients were enrolled and randomly assigned to receive L-carnitine (2000 mg/day) / placebo for 12 weeks. Micro RNA (MiR)-217 expression levels were assessed at the study baseline and at the end of study. The MiRNA-217 expression level were assessed in compare with 36 age, sex, and BMI matched NAFLD/NASH negative obese subjects, as control population.

Results

MiRNA-217 expression level was higher in NASH suffering group compared to NASH negative obese patients $(P<0.001)$. There was a significant difference in the expression level of MiRNA-217 between Lcarnitine and placebo receiving groups after the study intervention $(P=0.001)$; while the expression levels had no significant difference between the two groups at the initiation of the study. L-carnitine supplementation reduced MiRNA-217 expression levels in participants who were assigned to the supplementation group; while MiRNA-217 expression levels in the placebo received NASH, positive obese patients didn't change during the study.

Conclusion

MiRNA-217 expression level can be considered as a noninvasive practical biomarker for diagnosis, and treatment follow-up of NASH suffering patients. However, further studies are required to find out the exact relationships between MiRNA-217 expression and different stages of NAFLD.

Trial registration

This trial was registered on Iranian Clinical Trials Registry Platform IRCT201212052602N8 (https://en.irct.ir/trial/2379) on February 4, 2013.

\section{Background}


Non-alcoholic fatty liver disease (NAFLD) is characterized by fat accumulation in liver tissue which involves up to $74 \%$ of obese adults worldwide ${ }^{1,2}$. Nonalcoholic steatohepatitis (NASH) is a severe subtype of NAFLD with chronic necroinflammation and hepatocytes injury that is considered as progressive chronic liver disease and may be complicated by liver fibrosis, hepatic cirrhosis, liver failure and hepatocellular carcinoma ${ }^{3,4}$.

According to Multiple Parallel Hit hypothesis, NAFLD and its progression to NASH, cirrhosis and liver failure depend upon several factors including nutritional risk factors, lack of physical activity, insulin resistance, mitochondrial dysfunction, altered adipokines metabolism, oxidative stress, genetic susceptibility factors, epigenetic factors and lipotoxicity ${ }^{5,6}$.

Life style modification including nutrition counseling, gradual weight loss planning, and routine physical activity, in addition to pharmacologic interventions and surgical procedures are different therapeutic strategies to minimize obesity-induced $\mathrm{NASH}$ and its co-morbidities and challenges in patients ${ }^{7}$.

According to previous studies, Silent Information Regulator 1 (SIRT1) gene, which plays a critical role in hepatic lipid metabolism, reducing hepatic inflammation and oxidative stress, is considered as a potential therapeutic target in NAFLD suffering patients ${ }^{8}$. Previous studies demonstrated that microRNA-217 (MiRNA-217) inhibits SIRT1 expression and this inhibition may play an important role in the pathogenesis of NASH also ${ }^{9}$. On the other hand, L-carnitine plays a crucial role in fatty acids transportation thorough mitochondrial membrane and hepatic lipid metabolism and may have beneficial effects on NASH in obese patients ${ }^{10}$. Therefore, in the present study, we aimed to investigate the effect of L-carnitine on the expression of MiRNA-217 in obese NASH suffering patients.

\section{Methods}

\section{Patients and study design}

This parallel double-blind randomized controlled trial (RCT) was performed according to the guidelines of the Helsinki Declaration. This study was approved by the Ethics Committee of Tehran University of Medical Sciences (ethics committee reference number: 91-03-161-19463-69987) and was registered in the Iranian Clinical Trials Registry Platform with the reference code IRCT201212052602N8 (4/2/2013; https://en.irct.ir/trial/2379).

Written informed consent forms were signed by the patients before initiation of the study. The study was carried out in the nutrition clinic of Mashhad University of Medical Sciences (MUMS), from March 2013 to June 2014. Patients aged 18-65 years with Body Mass Index (BMI) $>25 \mathrm{Kg} / \mathrm{m}^{2}$ and definite diagnosis of $\mathrm{NASH}$ (Alanine Aminotransferase (ALT) $>3$ times more than the upper limit of the normal range and confirmatory ultrasonography outcomes), were eligible to be enrolled to the randomization process to receive supplement/placebo. Pregnancy, smoking, Insulin usage, receiving lipid-lowering drugs as well as steroidal and non-steroidal anti-inflammatory drugs, and antioxidant supplements, viral hepatitis, history 
of Cushing disease, hyperthyroidism and Wilson's disease were considered as exclusion criteria. All patients with eligibility criteria during the study period (71 subjects) were enrolled. At the same time, 36 age, sex, and BMI matched NAFLD/NASH negative obese subjects (by ALT test and ultrasonography findings) who were on weight loss program were enrolled and assigned to the control group. Venous blood samples were taken from all participant before and after intervention.

\section{Interventions and Randomization}

Firstly, all the participants were visited by an expert dietitian at the baseline of the study; a 3-day food recall was fulfilled by each participant to calculate his/her energy and nutrient intake and a $500 \mathrm{Kcal}$ deficit in daily caloric intake was applied to the recommended diet which was planned by the dietitian. Moreover, a 30-minute light to moderate exercise program for at least 3 days per week was recommended to all participants.

The randomization in assigning samples into the intervention or placebo receiving groups was performed based on a random numbers table which was computer generated by an independent research assistant. Sequentially numbered containers were used for the implementation of the random allocation sequence. All the participants and main researchers were blind to group allocation. Moreover, the study statistician was blind to group allocation prior to the analysis.

L-carnitine in form of $250 \mathrm{mg}$ tablets (Totally $2000 \mathrm{mg} /$ day, manufactured by [removed for blind peer review]) was administered to the patients who were assigned to the supplementation group for 12 weeks; while the placebo tablets were administered in similar shape, appearance and color to the placebo receiving group. Protocol adherence of each participant was applied by pill count strategy and consumption of $>80 \%$ of the administered supplement/placebo was considered as positive compliance to the treatment.

\section{Assessments}

Weight of patients was measured by a digital scale with minimum clothes and without shoes with an accuracy of 100 grams. Height was measured by an upstretched tape with a maximum error of 0.5 centimeters in a near-wall standing position. In order to assess miRNA expression, $5 \mathrm{~mL}$ of fasting intravenous blood was taken from each participant at the study baseline and at the end of study (12 weeks supplementation/placebo administration).

\section{MiRNA expression analysis}

Analysis of miRNA expression was performed by Relative quantitative real-time PCR using TaqMan prob technology. MicroRNAs was extracted from plasma samples using the mirvana paris kit (Ambion, AM1556) according to manufacturer's instruction. cDNAs were synthesized with MicroRNA-217 and MicroRNA-23b stem-loop primers, using AccuPower ${ }^{\circledR}$ CycleScript RT PreMix (Bioneer company, K-2046). Sequence of primers and probes are shown in table 1. Relative quantitative Real time PCR was carried out 
in 20ul reaction mixture containing 200 unit taq DNA polymerase enzyme and $0.25 \mathrm{uM}$ stem loop primers and probes. MicroRNA-23b was used as the reference MicroRNA. All reactions were performed as triplicate.

\section{Table 1. Primers, probe, and PCR product sequences for MiR-217 and MiR-23.}

\begin{tabular}{|ll|}
\hline Variable & Sequence \\
\hline MiR-217 sequence & UACUGCAUCAGGAACUGAUUGGA \\
\hline MiR-217-Stem-loop-RT & GTCGTATCCAGTGCAGGGTCCGAGGTATTCGCACTGGATCAGACTCCAAT \\
\hline MiR-217-Forward primer & TGCGCTACTGCATCAGGAACTG \\
\hline MiR-217-Reverse primer & CAGTGCAGGGTCCGAGGTA \\
\hline MiR-217-probe & TexasRed-ATTGGAGTCTGATCCAGTGCGAA-BHQ1 \\
\hline MiR -23 sequence & AUCACAUUGCCAGGGAUUACC \\
\hline MiR -23-RT- primer & GTCGTATCCAGTGCAGGGTCCGAGGTATTCGCACTGGATCAGACGGTAAT \\
\hline MiR -23 Forward primer & TGCGCATCACATTGCCAGGG \\
\hline MiR -23 Reverse primer & CAGTGCAGGGTCCGAGGTA \\
\hline MiR -23-probe & HEX-ATTACCGTCTGATCCAGTGCGAA-BHQ1 \\
\hline
\end{tabular}

\section{Statistical analysis}

The main purpose of our study was to evaluate whether L-carnitine supplementation had beneficial effects on the expression of miRNA-217 in obese patients with NASH. Therefore, the primary outcome was expression levels of miRNA-217. Chi-square or Fisher exact tests were used to compare the general characteristics of the subjects for qualitative variables. Paired t-test and Wilcoxon tests were used for intra-group comparisons of variables and Independent t-test and Mann-Whitney tests were used to compare the quantitative variables between the two groups.

Statistical analysis was calculated using SPSS Statistics version 22.0 (USA). Unpaired Student's t-test was used to compare overall expression of MicroRNA-217 levels between different groups. Fold change of MicroRNA-217 expression levels in all samples compared to their normal samples were calculated by using 2- $\Delta \Delta C T=2-(\Delta C T$ case $-\Delta C T$ normal $)$ formula ${ }^{11}$. Fold expression $>2$ was considered as increased expression and fold expression $<0.5$ was considered as decreased expression.

\section{Results}

After the eligibility assessment, 71 patients who met the inclusion criteria were randomly assigned to either the supplement or placebo groups. Of the 35 patients assigned to the placebo group, 5 patients were excluded due to unwillingness to continue their participation in the study. Finally, 36 patients 
received L-carnitine supplementation and 31 obese NASH positive patients received the placebo.

Moreover, 34 obese NASH negative control subjects were enrolled in the study, while two cases excluded following their unwillingness to participate continuation. The enrollment, randomization, and completion of the study outcomes are shown in Fig. 1.

According to the results of the present study, there was no statistically significant difference in age, weight, height, and gender of participants between the supplement, placebo receiving and control groups $(P>0.05)$. Table 2 shows the demographic characteristics of patients in the studied groups.

Table 2

Base line characteristics of the study participants.

\begin{tabular}{|lcccc|}
\hline $\begin{array}{l}\text { Group } \\
\text { Variable }\end{array}$ & $\begin{array}{l}\text { L-carnitine } \\
\text { received }\end{array}$ & $\begin{array}{l}\text { Placebo } \\
\text { received }\end{array}$ & $\begin{array}{l}\text { Matched } \\
\text { control }\end{array}$ & P-value \\
\hline Age (year) & $41.6 \pm 8.8$ & $45.3 \pm 12.1$ & $43.4 \pm 8.2$ & $0.3^{*}$ \\
\hline Male: N (\%) & $12(33)$ & $11(35)$ & $11(33)$ & $0.4^{* *}$ \\
\hline Height (Centimeters) & $167.6 \pm 9.7$ & $165.1 \pm 10.49$ & $162.7 \pm 8.7$ & $0.1^{*}$ \\
$\begin{array}{l}\text { Base line Weight } \\
\text { (Kilograms) }\end{array}$ & $89.4 \pm 14.7$ & $82.3 \pm 13.0$ & $82.2 \pm 18.1$ & $0.09^{*}$ \\
\hline $\begin{array}{l}\text { The data are presented as means } \pm \text { standard deviation; *: p-values were obtained from ANOVA test; **: } \\
\text { P-value was obtained from chi-squire test. }\end{array}$ & & & \\
\hline
\end{tabular}

Figure 2 depicts the relative MiRNA-217 expression levels in L-carnitine supplemented and placebo received groups during the study. As it is shown in Fig. 2, there was a significant difference in the expression level of MiRNA-217 between NASH positive and negative obese patients, as the expression level was higher in NASH suffering group $(P<0.001)$.

The results of our study showed that there was a significant difference in the expression level of MiRNA-

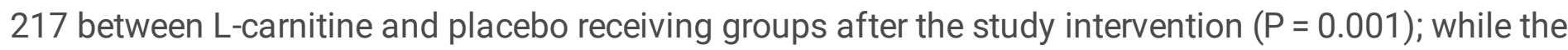
expression levels of this variable in two mentioned groups had no significant difference between the two groups before the intervention $(P=0.3)$. Table 3 shows the expression levels of MiRNA-217 in the Lcarnitine and placebo receiving groups during our study in compare with control group. 
Table 3

MiR-217 expression levels in two studied groups during the study.

\begin{tabular}{|llll|}
\hline $\begin{array}{l}\text { Group } \\
\text { Variable }\end{array}$ & $\begin{array}{l}\text { L-carnitine } \\
\text { supplemented }\end{array}$ & $\begin{array}{l}\text { Placebo } \\
\text { received }\end{array}$ & $\begin{array}{l}\text { P-value (Independent } \\
\text { T test) }\end{array}$ \\
\hline Base line MiR-217 expression level & $3.11 \pm 1.51$ & $2.25 \pm 1.63$ & 0.3 \\
\hline $\begin{array}{l}\text { MiR-217 expression level at the end } \\
\text { of the study }\end{array}$ & $1.07 \pm 1.02$ & $2.69 \pm 0.92$ & 0.001 \\
\hline \begin{tabular}{l} 
P-value (Paired T test) \\
\hline
\end{tabular} & $<0.001$ & 0.1 & - \\
\hline
\end{tabular}

As it is shown in Table 3, intra-group changes in serum expression levels of MiRNA-217 was not significant in the placebo received group during the study, while there was a significant reduction in the mean of MiRNA-217 expression level in the L-carnitine supplemented group $(P=0.1$, and $<0.001$, respectively).

Finally, changes of MiRNA-217 expression levels during the study were calculated by $2^{-\triangle \Delta C T}$ formula. In studying the mean \pm standard error of the mean (SEM) of MiRNA-217 expression changes, it was $2.07 \pm$ 0.4 in the supplemented group which was statistically different from the placebo received group (mean \pm SEM: $-5.6 \pm 0.5, P=<0.001$ ). Relative MiRNA-217 expression levels in two studied group during the study are shown in Fig. 3.

\section{Discussion}

To the best of our knowledge, although previous studies demonstrated several alterations in some MicroRNAs expression levels, including MiR-21, MiR-34a, MiR-122, and MiR-451 at different stages of NAFLD disease; the present study is the first study investigating L-carnitine supplementation effects on the expression level of MiRNA-217 in NASH suffering patients with obesity ${ }^{12-15}$.

Given that MiRNA-217 may affect the pathogenesis of NASH, by modulating of SIRT1 expression levels; the results of our study demonstrated an increased expression level of this MiR in the serum of NASH positive patients ${ }^{8,9}$. Moreover, L-carnitine supplementation reduced MiRNA-217 expression levels in participants who were assigned to the supplementation group; while MiRNA-217 expression levels in the placebo received NASH, positive obese patients didn't change during the study. Therefore, the results of the present study suggest that MiRNA-217 expression level can be considered as a noninvasive practical biomarker for diagnosis, and treatment follow-up of NASH suffering patients.

Further, the results of our study were consistent to previous studies reported beneficial effects of Lcarnitine supplementation on oxidative stress, NAFLD/NASH, and liver fibrosis ${ }^{10,16,17}$. According to previous studies, a major cause of fat accumulation and oxidative stress exacerbation in hepatic tissue, is inhibition of mitochondrial fatty acid oxidation which may be modified by L-carnitine supplementation 17, 18. Moreover, it is reported that L-carnitine supplementation may lead to significant decrease in 
inflammation and oxidative stress levels, elevation of caspase-2 protein expression (an anti-fibrotic agent), and reduction of necrosing factor (NF) KB p65 also ${ }^{17}$.

As it is reported in previous studies, SIRT1 expression in NAFLD-induced high-fat diet mice was significantly decreased ${ }^{19}$. SIRT1, the crucial regulator of autophagy and lipogenesis, regulates lipid homeostasis by regulating PPAR a (peroxisome proliferators activated receptor $\mathrm{a}$ ); and hepatocytespecific repression of SIRT1 impairs PPAR a signaling and consequently, decreases beta-oxidation of fatty acids ${ }^{20}$. On the other hand, Lipin 1 , one of SIRT1 downstream genes in which its acetylation is impaired by expression alterations of SIRT1, is a key regulator of lipid metabolism in hepatocytes and its expression was significantly increased in alcohol-induced NAFLD mice ${ }^{21}$. MiRNA-217 is an endogenous inhibitor of the SIRT1 gene, binding to the 3 -UTR region and interference with the translation and therefore expression of SIRT $1{ }^{22}$. In fact, MiRNA-217's deleterious effect on lipin1 and depletion of SIRT1 eventually lead to fat accumulation and alcohol-dependent steatohepatitis ${ }^{23}$.

Another mechanism that researchers have proposed for the function of MiRNA-217 is the effect of it through the PTEN (phosphatase and tensin homolog) gene as one of the inhibitors of AKT (Protein kinase B) gene activity; which is considered as an important gene that plays role in activating liver stellate cells (the main stimulus of liver fibrosis), thereby increasing the levels of MiRNA-217 will may stimulate liver fibrosis 24,25 .

According to the results of this double-blind RCT, MiRNA-217 expression level may be a simple practical biomarker for NASH diagnosis and follow up; however, further long-term large population-based studies are required to find out the exact relationships between MiRNA-217 expression levels, its determinants, and consequent genomics and epigenetics outcomes and different stages of NAFLD. The major findings of the present study as well as important methodological points are presented as graphical abstract in Fig. 4.

Impossibility of the liver biopsy as the gold standard of NAFLD/NASH diagnosis due to its invasive modality, and not assessing of carnitine levels in the study participants were two important limitations of our study. Additionally, non-assessing of SIRT1 expression levels was another limitation of the present study.

\section{Conclusion}

L-carnitine supplementation reduced MiRNA-217 expression levels in participants who were assigned to the L-carnitine supplementation group; while MiRNA-217 expression levels in the placebo received NASH, positive obese patients didn't change during 12 weeks follow up.

The results of our study highlight the importance of serum MiR-217 expression level as a candidate biomarker to be considered in NASH diagnosis and treatment approaches. However, further studies are 
required to find out the exact relationships between MiRNA-217 expression and different stages of NAFLD.

\section{Abbreviations}

NASH, Nonalcoholic steatohepatitis; NAFLD, Non-alcoholic fatty liver disease; SIRT1, Silent information regulator 1; RCT, randomized controlled trial; BMI, Body mass index; ALT, Alanine Aminotransferase; MiRNA, microRNA; PTEN, phosphatase and tensin homolog; PPAR a, peroxisome proliferators activated receptor $\mathrm{a} ; \mathrm{NF}$, necrosing factor.

\section{Declarations}

\section{Ethics approval and consent to participate}

This double-blind randomized controlled trial (RCT) was approved by the Ethics Committee of Tehran University of Medical Sciences (ethics committee reference number: 91-03-161-19463-69987) and was registered in the Iranian Clinical Trials Registry Platform with the reference code IRCT201212052602N8 (https://en.irct.ir/trial/2379).

Written informed consent forms were signed by the patients before initiation of the study.

\section{Consent for publication}

Not applicable.

\section{Availability of data and materials}

All data generated or analyzed during this study are included in the manuscript. The study data is available from the corresponding author upon reasonable request.

\section{Competing interests}

The authors report no conflicts of interest for this work.

\section{Funding}

This research was financially supported by Tehran University of Medical Sciences, and deputy of research, Mashhad University of Medical Sciences, Mashhad, Iran (grant number: 920585).

\section{Authors' contributions}

$\mathrm{FI}, \mathrm{SA}, \mathrm{MA} 1, \mathrm{MM}$, and MN contributed to the conception and design of the study. SA, FI, and MA2 participated in data collection from the participants and laboratory analysis. MS, FR, SA, and MM 
supported the analysis and interpretation. MN, MM, and MA1 supervised the study. FR, FI, and MM participated in drafting and editing the manuscript. All authors read and approved the final manuscript.

[1: Mohsen Azimi-Nezhad; 2: Mahsa Asle-Mohajeri]

\section{Acknowledgments}

We are very thankful to numerous colleagues with whom we have shared our research and all the patients who participated in this study.

\section{References}

1. Rolo AP, Teodoro JS, Palmeira CM. Role of oxidative stress in the pathogenesis of nonalcoholic steatohepatitis. Free Radical Biology and Medicine. 2012;52(1):59-69.

2. Chalasani N, Younossi Z, Lavine JE, et al. The diagnosis and management of non-alcoholic fatty liver disease: practice Guideline by the American Association for the Study of Liver Diseases, American College of Gastroenterology, and the American Gastroenterological Association. Hepatology. 2012;55(6):2005-23. doi:10.1002/hep.25762.

3. Takaki A, Kawai D, Yamamoto K. Molecular mechanisms and new treatment strategies for nonalcoholic steatohepatitis (NASH). International journal of molecular sciences. 2014;15(5):7352-79.

4. Angulo P. Nonalcoholic fatty liver disease. N Engl J Med. 2002;346(16):1221-31. doi:10.1056/NEJMra011775.

5. Buzzetti E, Pinzani M, Tsochatzis EA. The multiple-hit pathogenesis of non-alcoholic fatty liver disease (NAFLD). Metabolism. 2016;65(8):1038-48. doi:10.1016/j.metabol.2015.12.012.

6. Fang $Y-L$, Chen $H$, Wang $C-L$, Liang L. Pathogenesis of non-alcoholic fatty liver disease in children and adolescence: From "two hit theory" to "multiple hit model". World journal of gastroenterology. 2018;24(27):2974-83. doi:10.3748/wjg.v24.i27.2974.

7. Mintziori G, Polyzos SA. Emerging and future therapies for nonalcoholic steatohepatitis in adults. Expert Opin Pharmacother. 2016;17(14):1937-46. doi:10.1080/14656566.2016.1225727.

8. Ding R-B, Bao J, Deng C-X. Emerging roles of SIRT1 in fatty liver diseases. International journal of biological sciences. 2017;13(7):852-67. doi:10.7150/ijbs.19370.

9. Menghini R, Casagrande V, Cardellini M, et al. MicroRNA 217 modulates endothelial cell senescence via silent information regulator 1. Circulation. 2009;120(15):1524.

10. Moghadam SA, Nematy M, Eghtesadi S, et al. Effects of L-carnitine supplementation on inflammatory factors and malondialdehyde in patients with nonalcoholic steatohepatitis (NASH). Current Topics in 
Nutraceutical Research. 2015;13:135-41.

11. Schmittgen TD, Livak KJ. Analyzing real-time PCR data by the comparative CT method. Nature Protocols. 2008;3(6):1101-8. doi:10.1038/nprot.2008.73.

12. Baranova A, Maltseva D, Tonevitsky A. Adipose may actively delay progression of NAFLD by releasing tumor-suppressing, anti-fibrotic miR-122 into circulation. Obesity reviews. 2019;20(1):108-18.

13. Lin B, Ma Y, Wu S, Liu Y, Liu L, Wu L. Novel serum biomarkers for noninvasive diagnosis and screening of nonalcoholic fatty liver disease-related hepatic fibrosis. Omics: a journal of integrative biology. 2019;23(4):181-9.

14. Musaddaq G, Shahzad N, Ashraf MA, Arshad MI. Circulating liver-specific microRNAs as noninvasive diagnostic biomarkers of hepatic diseases in human. Biomarkers. 2019;24(2):103-9.

15. Ando $\mathrm{Y}$, Yamazaki $\mathrm{M}$, Yamada $\mathrm{H}$, et al. Association of circulating miR-20a, miR-27a, and miR-126 with non-alcoholic fatty liver disease in general population. Scientific reports. 2019;9(1):1-8.

16. Somi MH, Fatahi E, Panahi J, Havasian MR, Judaki A. Data from a randomized and controlled trial of LCarnitine prescription for the treatment for Non- Alcoholic Fatty Liver Disease. Bioinformation. 2014;10(9):575-9. doi:10.6026/97320630010575.

17. Mollica G, Senesi P, Codella R, et al. L-carnitine supplementation attenuates NAFLD progression and cardiac dysfunction in a mouse model fed with methionine and choline-deficient diet. Digestive and Liver Disease. 2020;52(3):314-23. doi:10.1016/j.dld.2019.09.002.

18. Brunt EM. Pathology of nonalcoholic fatty liver disease. Nature reviews Gastroenterology \& hepatology. 2010;7(4):195-203.

19. Deng XQ, Chen LL, Li NX. The expression of SIRT1 in nonalcoholic fatty liver disease induced by highfat diet in rats. Liver International. 2007;27(5):708-15.

20. Rahman S, Islam R. Mammalian Sirt1: insights on its biological functions. Cell Communication and Signaling. 2011;9(1):11.

21. Yin $H$, Hu M, Zhang R, Shen Z, Flatow L, You M. MicroRNA-217 promotes ethanol-induced fat accumulation in hepatocytes by down-regulating SIRT1. J Biol Chem. 2012;287(13):9817-26. doi:10.1074/jbc.M111.333534.

22. Gjorgjieva M, Sobolewski C, Dolicka D, de Sousa MC, Foti M. miRNAs and NAFLD: from pathophysiology to therapy. Gut. 2019;68(11):2065-79.

23. Shen Z, Ajmo JM, Rogers CQ, et al. Role of SIRT1 in regulation of LPS- or two ethanol metabolitesinduced TNF-alpha production in cultured macrophage cell lines. Am J Physiol Gastrointest Liver Physiol. 
2009;296(5):G1047-53. doi:10.1152/ajpgi.00016.2009.

24. Xia H, Ooi LL, Hui KM. MicroRNA-216a/217-induced epithelial-mesenchymal transition targets PTEN and SMAD7 to promote drug resistance and recurrence of liver cancer. Hepatology. 2013;58(2):629-41. doi:10.1002/hep.26369.

25. Sun J, Li ZP, Zhang RQ, Zhang HM. Repression of miR-217 protects against high glucose-induced podocyte injury and insulin resistance by restoring PTEN-mediated autophagy pathway. Biochem Biophys Res Commun. 2017;483(1):318-24. doi:10.1016/j.bbrc.2016.12.145.

\section{Figures}
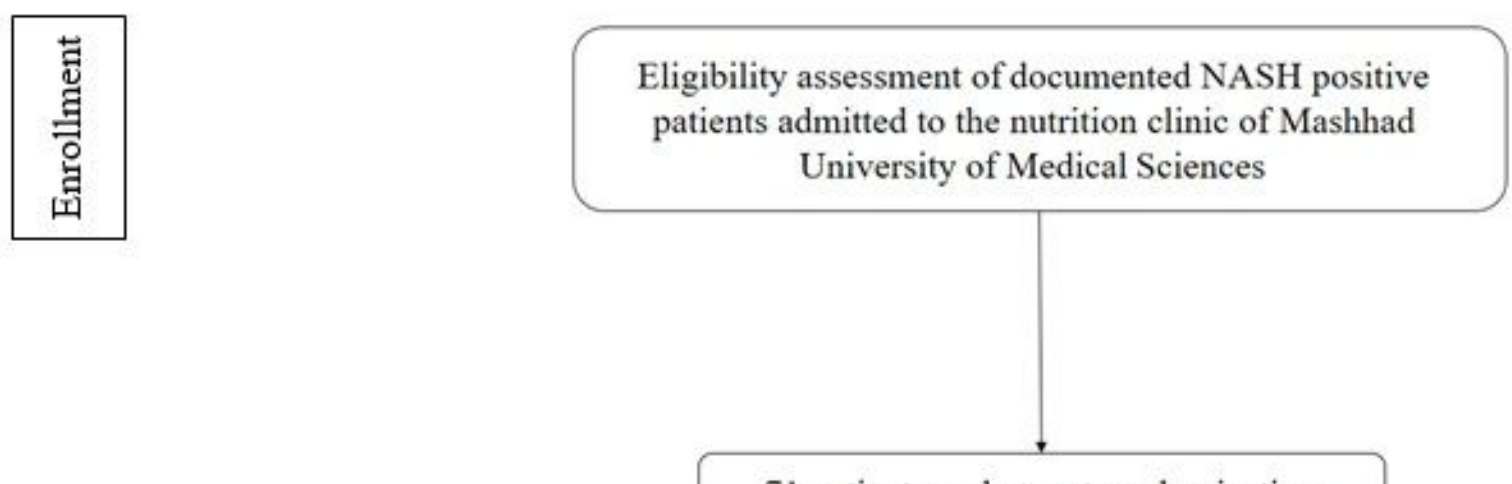

71 patients underwent randomization

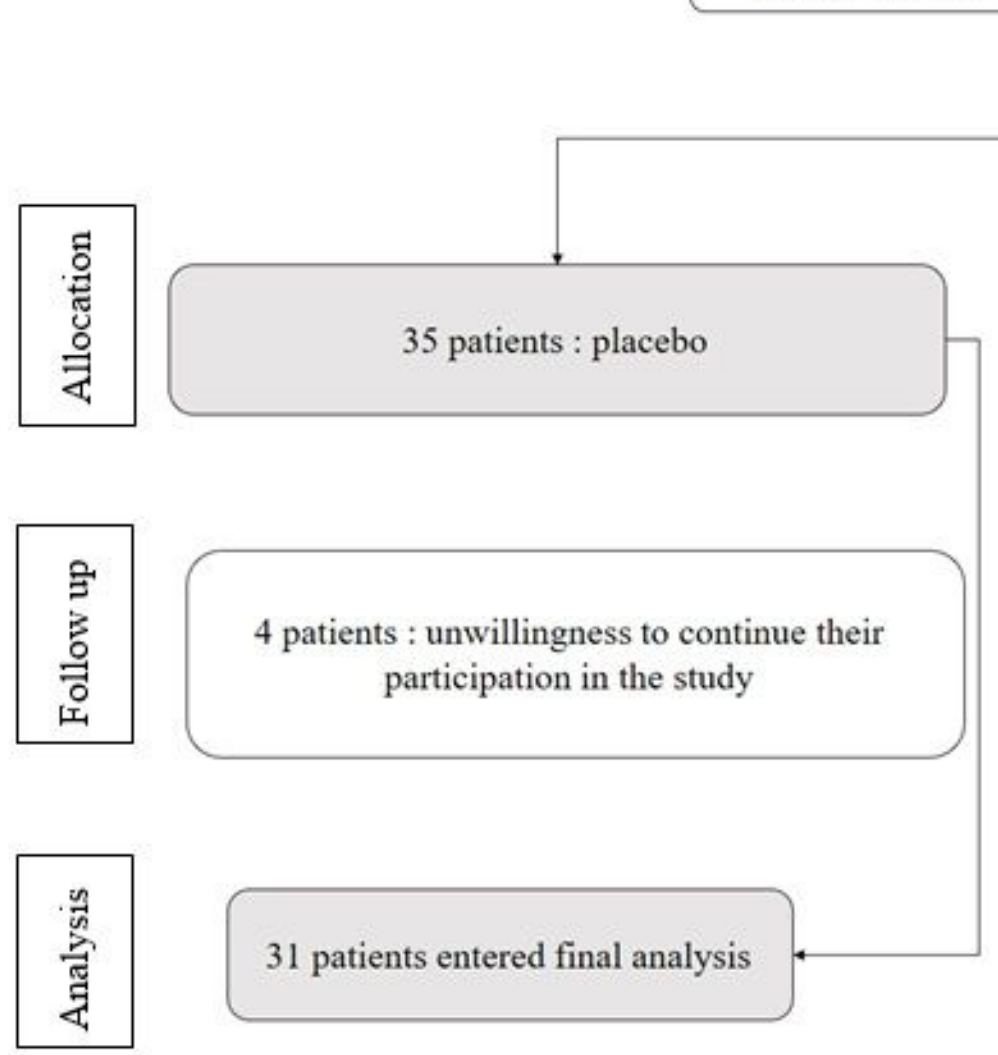

36 patients : L-carnitine at a dose of $2000 \mathrm{mg} /$ day

36 patients entered final analysis

\section{Figure 1}


Flowchart of the study.

3.5

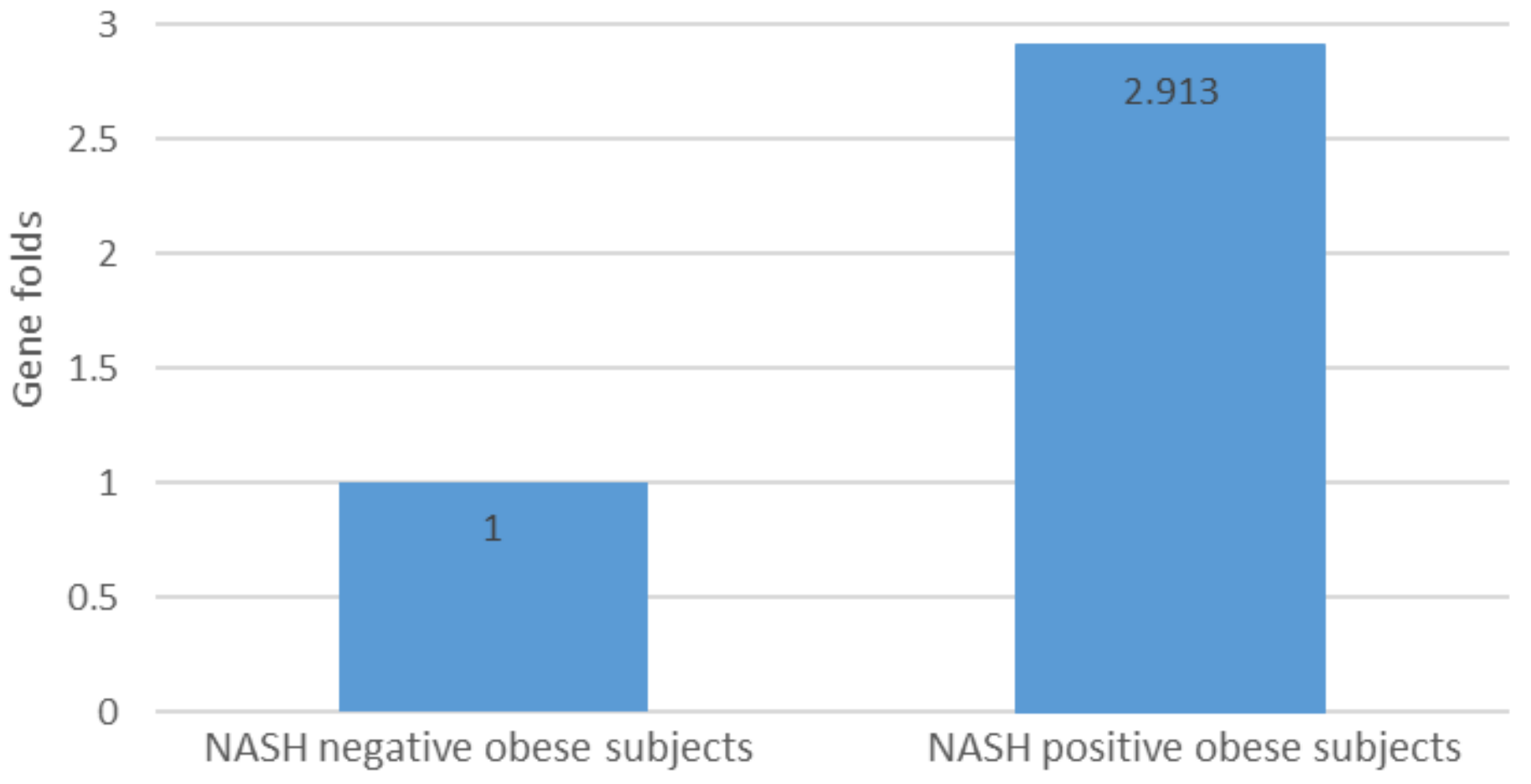

Figure 2

Relative MiR-217 expression levels in NASH positive and negative obese participants at baseline of the study. 
1.2

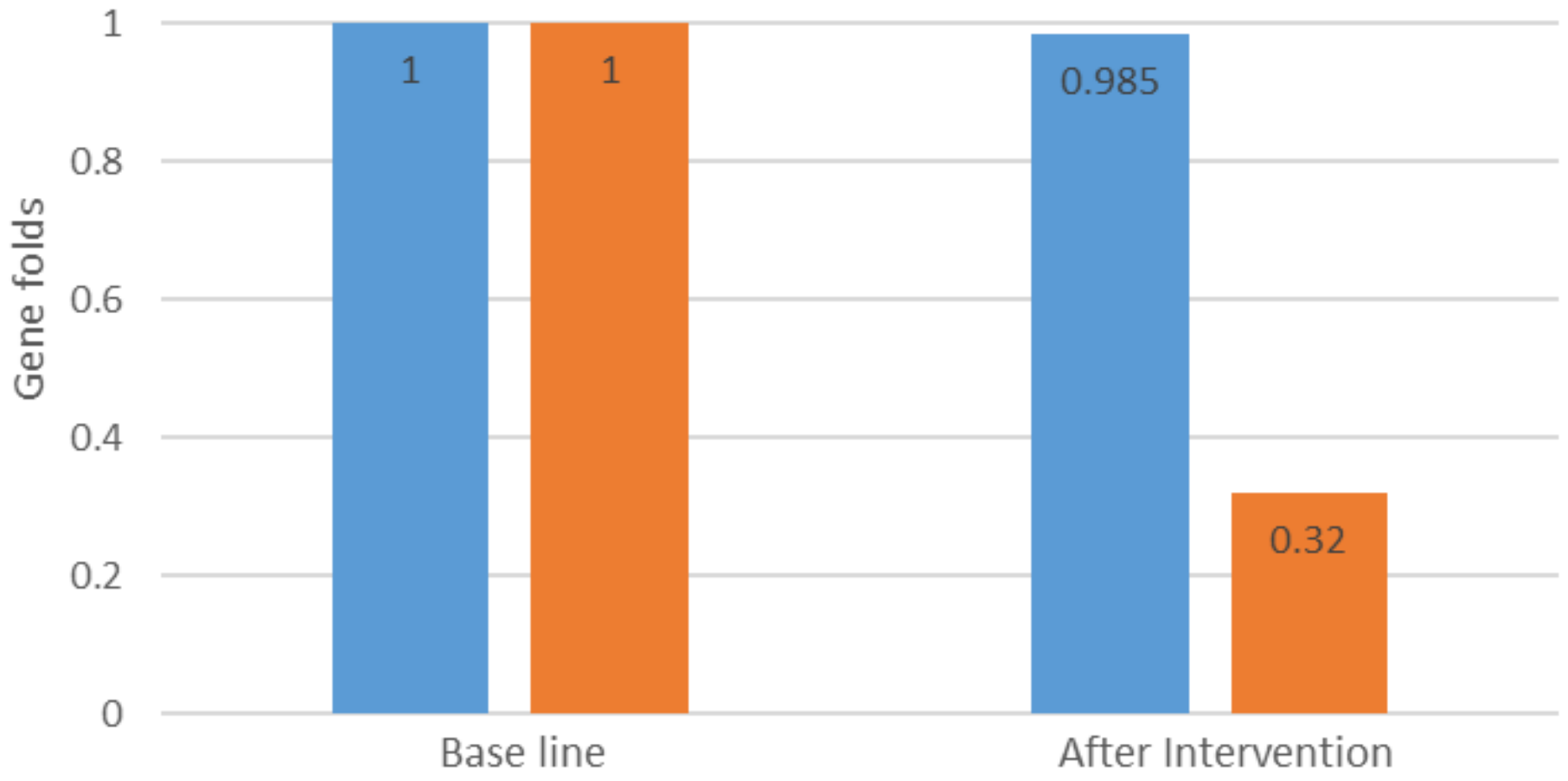

Figure 3

Relative MiR-217 expression levels in L-carnitine supplemented and placebo received groups during the study; (L-carnitine supplemented group, placebo received group). 
Patients :

18-65 years;

Body Mass Index $(\mathrm{BMI})>25 \mathrm{Kg} / \mathrm{m}^{2}$
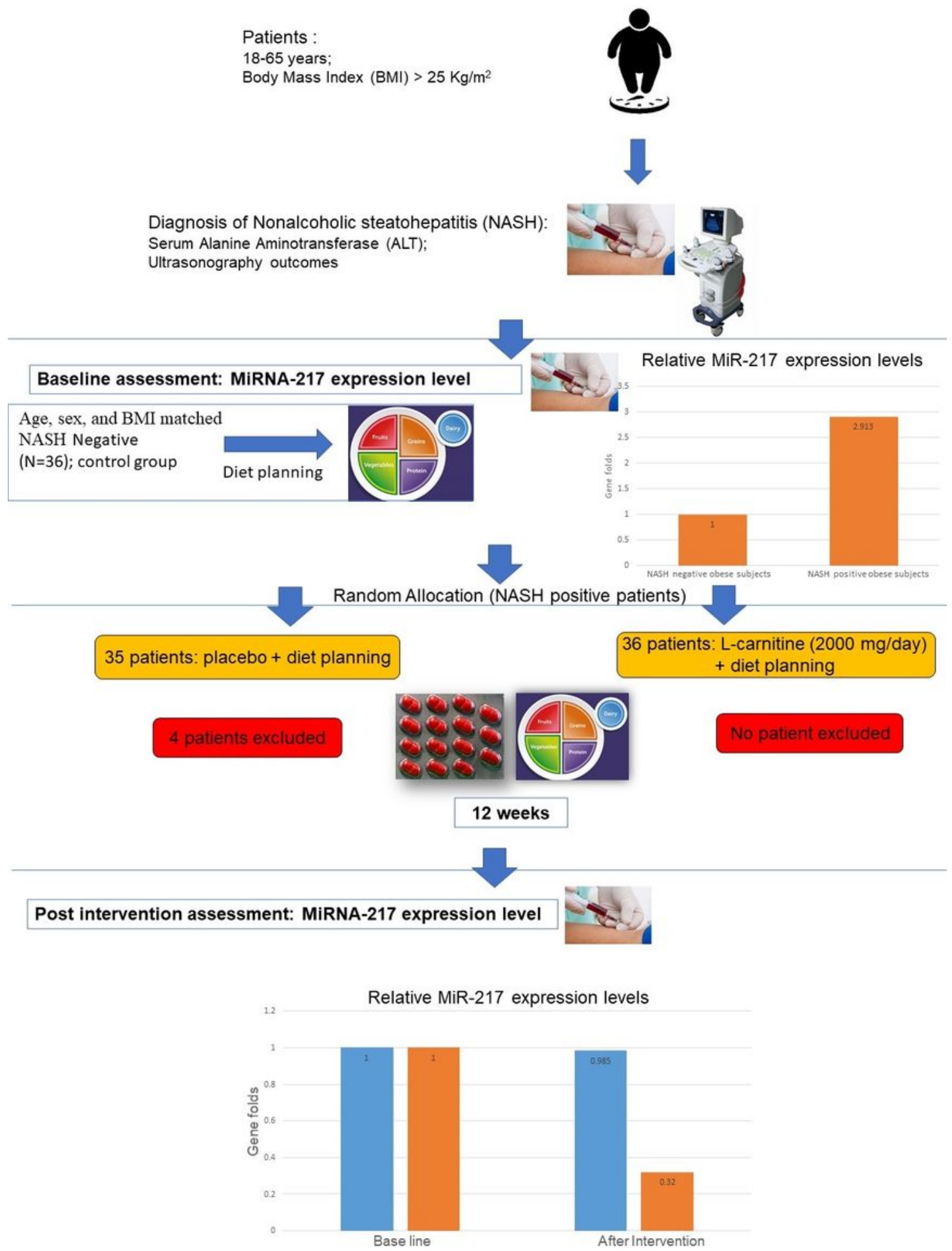

\section{Figure 4}

Graphical abstract. 